\title{
MALIGNANT COLO-DUODENAL FISTULA: A CASE REPORT AND LITERATURE REVIEW
}

\author{
Md Nur Hossain Bhuiyan ${ }^{1}$ Srikanta Banik ${ }^{2}$ Omor Faruk Tuhin ${ }^{3}$ Syed Md Muhsin ${ }^{2}$ \\ Saiful Haque ${ }^{1} \mathrm{~S}$ M Ashraf $\mathrm{Ali}^{4}$ Omor Faruque Yusuf ${ }^{5}$
}

\begin{abstract}
Malignant colo-duodenal fistula is a rare complication of malignant bowel disease, difficult to diagnose clinically. The case was diagnosed by colonoscopy and barium enema and the patient was treated conservatively because of inoperability and poor general condition. A high index of clinical suspicion about the condition may help early diagnosis and curative treatment.
\end{abstract}

Key words: colo-duodenal fistula; malignant; clinical suspicion

\section{Introduction}

Malignant colo-duodenal fistula is a rare complication of right sided colonic cancer. The first known case was reported in 1862 by Halden ${ }^{1}$. Hershenson reported $1 / 8100$ in autopsies ${ }^{2}$. Izumi reviewed 34 cases in $\mathrm{Japan}^{3}$. The disease may remain asymptomatic for prolonged period of time. Often becomes symptomatic with manifestations of primary colonic carcinoma, features related to fistula or metastatic deposites ${ }^{4}$. Classical symptoms; upper abdominal pain, loose motion, faeculant vomiting are often absent ${ }^{5}$. Radiology is useful to delineate the fistula as the difference in surgical management of gastro-colic and a duodeno-colic fistula is profound. CT scan is of great value in assessing metastatic spread and local invasion of the primary lesion $^{6}$. Management is very difficult and should be a multidisciplinary approach.

\section{Case Presentation}

Mr Yakub 50 years, a service holder from southern Chittagong presented with a lump in the right hypochondrium for two years, alteration of

\footnotetext{
1. Assistant Professor of Surgery Chittagong Medical College, Chittagong

2. Post graduate (MS Surgery, Thesis part) student Chittagong Medical College, Chittagong

3. Post graduate (FCPS Surgery- 2nd part) student Chittagong Medical College, Chittagong

4. Associate Professor of Surgery Chittagong Medical College, Chittagong

5. Professor of Surgery Chittagong Medical College, Chittagong
}

Correspondence : Dr Md Nur Hossain Bhuiyan bowel habit, gross weight loss and anorexia for last one and half years. He was having persistent diarrhoea for 3 months. The patient had an attack of cerebrovascular accident two month back. The patient was severely anemic and malnourished. Abdominal examination revealed a firm, mildly tender lump in the right hypochondriac region about $10 \times 8 \mathrm{~cm}$ in diameter. No other organomegaly was detected. Colonoscopy showed a growth in the ascending colon, hepatic flexure and proximal transverse colon. Histopathology report was poorly differentiated adenocarcinoma. A coloduodenal fistula was demonstrated by Barium enema. Upper gastro- intestinal tract endoscopy reveled onward narrowing of duodenum from its bulb. Ultrasonography showed a possible colonic mass and abdominal lymphadenopathy. CT scan of brain reveals metastatic deposit. CEA level was 154 $\mathrm{ng} / \mathrm{ml}$. and chest X-ray was normal.

He was diagnosed as a case of malignant coloduodenal fistula with cerebrovascular disease with left sided hemiplegia. A multidisciplinary meeting recommended supportive treatment for the patient. The patient succumbed to his disease 15 days after discharge from hospital.

\section{Discussion}

It is unusual, however, for colon cancers to invade the duodenum to such an extent that a malignant fistula is created. The first case report of a colo-duodenal fistula was by Haldane in $1862^{1}$. His patient had a malignant fistula arising from the hepatic flexure... Hershenson documented only one case among 8,100 autopsies ${ }^{2}$. Observation of 1400 cases by Hakarmi M et al detected only two malignant colo-duodenal fistulae $^{7}$. In the United States one report showed the incidence of duodenocolic fistula is 1 in 900 colorectal carcinomas ${ }^{8}$. One article reported two cases, both of which had synchronous cancers that had to be removed en-bloc ${ }^{9}$.

In our case the patient presented with a lump. No specific symptoms related to coloduodenal fistula detected. Patients with malignant duodenal fistulae can present with symptoms from the primary colonic carcinoma, from the fistula or from metastatic 
disease ${ }^{10}$. The fistula often results in diarrhoea and vomiting with dramatic weight loss. Upper abdominal pain is usually present as is general malaise both from the presence of the disease and from the metabolic sequelae it causes ${ }^{10-13}$. The diarrhoea relates to colonic bacterial contamination of the upper intestines rather than to a pure mechanical effect ${ }^{14}$. It has also been suggested that duodenal bile salts have an irritating effects on colonic mucosa resulting in diarrhoea ${ }^{15}$. Vomiting may be faeculant or truly faecal with foul smelling eructation; but in some case reports this 'classic' symptomatology was often absent despite a fistula being present and patent enough to allow barium through $\mathrm{it}^{16}$. Occasionally patients will present with a gastro-intestinal bleeding.

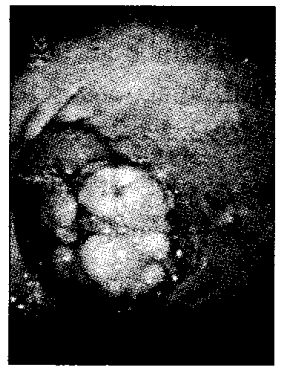

Fig 1 : Showing single nodular mass in the third part of the duodenum

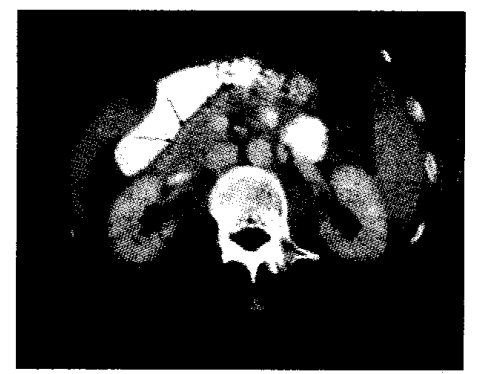

Fig 2: Showing CT of the abdomen detecting a mass in the duodenum

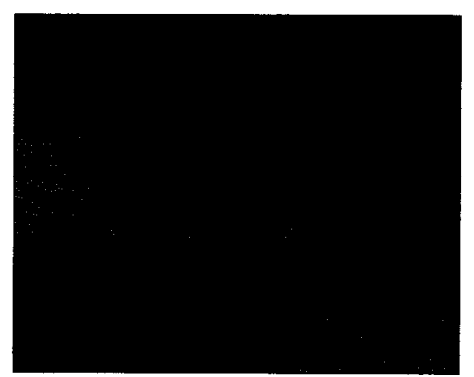

Fig 3 : Histopathological picture of resected duodenal tumour
We diagnosed the case by Barium enema, showed abnormal communication between right side of the colon and second part of the duodenum. Radiology is useful to delineate the fistula as the difference in surgical management between a gastro-colic and a duodeno-colic fistula is profound. Barium enema seemed more likely to delineate the fistula than barium meals ${ }^{17}$. Now a days CT scanning is of great value in assessing metastatic spread as well as assessing the local invasion of the primary ${ }^{18}$.

We treated the case conservatively as the general condition and disease status was not suitable for surgery. Treatment of malignant colo-duodenal fistulae depends on the extent of the primary tumour, the presence of metastatic disease and the general condition of the patient ${ }^{19}$. It is often necessary to spend time to rehydrate and transfusing the patient and correcting the electrolyte disturbances. Some patients present with gross weight loss from malnutrition. Malnutrition is due to the malabsorptive state results from bacterial overgrowth in the small bowel ${ }^{20}$. These patients may be benefited from pre-operative total parenteral nutrition (TPN). Other authors advocate surgery as soon as feasible.

There are various curative operations reported, all of which include a right hemicolectomy. Chang et.al treated 20 of his patients with right hemicolectomy with partial duodenectomy and primary closure of the duodenal wall defect ${ }^{21}$. His mortality rate was $28 \%$, mainly attributed to leakage from the duodenal defect and local recurrence. Ellis described using a jejunal loop to close the duodenal wall defect ${ }^{22}$ Colo-duodenal fistulas are associated with advanced carcinomas of the hepatic flexure. Recent times, these fistulae rarely occur because of an earlier detection and resection of the tumour ${ }^{23}$.

Izumi reviewed a series of 34 cases of malignant colo-duodenal fistulae in Japan, and their survival with en bloc pancreaticoduodenectomy ranged from 7 days to 4 years (median survival was 10 months, male female ratio was $15: 19^{3}$ reported on a patient following the two-stage. The standard management protocols of colo-duodenal fistula are-Curative treatment in the form of right hemicolectomy with pancreaticoduodenectomy. Palliative treatment, feeding jejunostomy with ileostomy or right hemicolectomy with partial duodenectomy is an alternative $^{24}$. Average mean survival after curative treatment ranges from 1-2.5 years and with palliative 
disease ${ }^{10}$. The fistula often results in diarrhoea and vomiting with dramatic weight loss. Upper abdominal pain is usually present as is general malaise both from the presence of the disease and from the metabolic sequelae it causes ${ }^{10-13}$. The diarrhoea relates to colonic bacterial contamination of the upper intestines rather than to a pure mechanical effect ${ }^{14}$. It has also been suggested that duodenal bile salts have an irritating effects on colonic mucosa resulting in diarrhoea ${ }^{15}$. Vomiting may be faeculant or truly faecal with foul smelling eructation; but in some case reports this 'classic' symptomatology was often absent despite a fistula being present and patent enough to allow barium through $\mathrm{it}^{16}$. Occasionally patients will present with a gastro-intestinal bleeding.

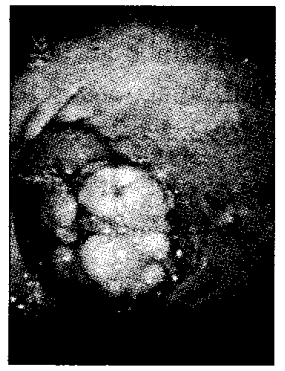

Fig 1 : Showing single nodular mass in the third part of the duodenum

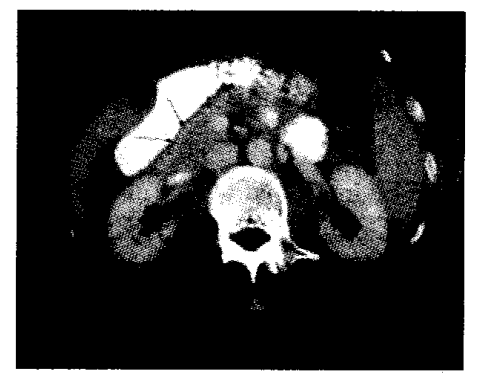

Fig 2: Showing CT of the abdomen detecting a mass in the duodenum

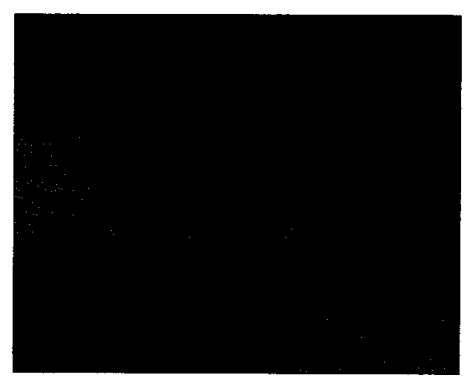

Fig 3 : Histopathological picture of resected duodenal tumour
We diagnosed the case by Barium enema, showed abnormal communication between right side of the colon and second part of the duodenum. Radiology is useful to delineate the fistula as the difference in surgical management between a gastro-colic and a duodeno-colic fistula is profound. Barium enema seemed more likely to delineate the fistula than barium meals ${ }^{17}$. Now a days CT scanning is of great value in assessing metastatic spread as well as assessing the local invasion of the primary ${ }^{18}$.

We treated the case conservatively as the general condition and disease status was not suitable for surgery. Treatment of malignant colo-duodenal fistulae depends on the extent of the primary tumour, the presence of metastatic disease and the general condition of the patient ${ }^{19}$. It is often necessary to spend time to rehydrate and transfusing the patient and correcting the electrolyte disturbances. Some patients present with gross weight loss from malnutrition. Malnutrition is due to the malabsorptive state results from bacterial overgrowth in the small bowel ${ }^{20}$. These patients may be benefited from pre-operative total parenteral nutrition (TPN). Other authors advocate surgery as soon as feasible.

There are various curative operations reported, all of which include a right hemicolectomy. Chang et.al treated 20 of his patients with right hemicolectomy with partial duodenectomy and primary closure of the duodenal wall defect ${ }^{21}$. His mortality rate was $28 \%$, mainly attributed to leakage from the duodenal defect and local recurrence. Ellis described using a jejunal loop to close the duodenal wall defect ${ }^{22}$ Colo-duodenal fistulas are associated with advanced carcinomas of the hepatic flexure. Recent times, these fistulae rarely occur because of an earlier detection and resection of the tumour ${ }^{23}$.

Izumi reviewed a series of 34 cases of malignant colo-duodenal fistulae in Japan, and their survival with en bloc pancreaticoduodenectomy ranged from 7 days to 4 years (median survival was 10 months, male female ratio was $15: 19^{3}$ reported on a patient following the two-stage. The standard management protocols of colo-duodenal fistula are-Curative treatment in the form of right hemicolectomy with pancreaticoduodenectomy. Palliative treatment, feeding jejunostomy with ileostomy or right hemicolectomy with partial duodenectomy is an alternative $^{24}$. Average mean survival after curative treatment ranges from 1-2.5 years and with palliative 
treatment it will reduce to 4 months to 1 year ${ }^{25}$.

\section{Conclusion}

Coloduodenal fistulas from colonic primaries are rare but important to identify preoperatively for proper management. Treatment of malignant coloduodenal fistula depends upon extent of primary tumour, metastatic deposit and general condition of the patients as en bloc resection with curative intent may well require a pancreaticoduodenectomy. The importance of accurate staging and repeated clinical examination along with a high index of suspicion cannot be over-emphasized.

\section{References}

1. Haldane DR: Case of cancer of the caecum, accompanied by with caecoduodenal and caecocolic fistulae. Edinburgh Med J 1862; 7: 624-629

2. Hershenson LM, Kirsner JB: Duodeno-colic fistula. Gastroenterology 1951; 19: 864-873

3. Izumi Y, Ueki T, Naritomi G, Akashi Y, Miyoshi A, Fukuda T: Malignant duodenocolic fistula: report of a case and considerations for operative management.Surg Today 1993; 23: 920-925

4. Calmenson M, Black BM: Surgical management of carcinoma of the right portion of the colon. Surgery $1947 ; 21: 476-481$

5. Mortensen NJ, Nicholls RJ, Northover JMA, Williams NS: The colon, rectum and anus. In Companion of Surgical Studies. Second edition. Edited by: Burnand KG, Young AE. London: Churchill Livingstone; 1999; 813-876

6. Welch JP, Warshaw AL: Malignant duodenocolic fistulas. Am J Surg 1977, 133:658-661. Iuchtman M, Zer M, Plavnick Y, Rabinson S: Malignant duodeno-colic fistula: the role of extended surgery. $J$ Clin Gastroenterol 1993; 16: 22-25

7. Hakarmi M, Dadgostar D, Feshareki R: Invasive carcinoma of the colon and duodenum with duodeno-colic fistula: report of a case.Am J Proctol 1976; 27: 35-36

8. Raffensperger EC, Sylvester LE, Ferguson LK: Duodeno-colic fistula due to carcinoma of the hepatic flexure; report of a case with a discussion of the surgical problems encountered. Ama Arch Surg 1957; 74: 333-337
9. Vieta JO, Blanco R, Valentini GR: Malignant duodeno-colic fistula: report of two cases, each with one or more synchronous gastrointestinal cancers. Dis Col Rectum 1976; 19: 542-552

10. Calvert DG, Medhurst GA: Fistula formation between the duodenum and colon. A report of three cases and a review of the condition. BJS 1960; 48: 136-139

11. Medhurst GA: Duodeno-colic fistula. $\mathrm{Br} \mathbf{J}$ Radiol 1956; 29: 381-385

12. Ergin MA, Alfonso A, Auda SP, Waxman M: Primary carcinoma of the duodenum producing a malignant duodeno-colic fistula. Dis Col Rectum 1978; 21: 408-412

13. Reissman P, Steinhagen RM, Enright PF: Duodeno-colic fistula: an unusual presentation of esophageal squamous cell carcinoma. Mt Sinai J Med 1992; 59: 75-78

14. Guzzetti A: A case of duodenocolic fistula caused by duodenal ulcer. Chir Ital 1981; 33: 934-937

15. Smith TR, Gouldin RR: Radiographic and clinical sequelae of the duodenocolic anatomic relationship: two cases of Crohn's disease with fistulization to the duodenum. Dis Col Rectum $1977 ; 20: 257-262$

16. 16. Korelitz BI: Colonic-duodenal fistula in Crohn's disease. Am J Dig Dis 1977; 22:10401048

17. Glass RE: Internal fistulas in Crohn's disease. Dis Col Rectum 1985; 28: 557-561

18. Pichney LS, Fantry GT, Graham SM: Gastrocolic and duodeno-colic fistulas in Crohn's disease. J Clin Gastroenterol 1992; 15: 205-211

19. Poggioli G: Duodenal involvement of Crohn's disease: three different clinicopathologic patterns.Dis Col Rectum 1977; 40: 179-183

20. Neville WE: Duodeno-colic fistula due to acute cholecystitis.Am J Surg 1953; 87: 300-302

21. Chang AE, Rhoads JE: Malignant duodenocolic fistulas: a case report and review of the literature. J Surg Oncol 1982; 21: 33-36

22. Ellis H, Morgan MN, Wastell C: 'Curative' surgery in carcinoma of the colon involving duodenum. A report of six cases. BJS 1972; 59: 932-935 
23. Edmunds PK, Harvard C: Duodeno-colic fistula due to gallstones. BJS 1961; 49: 253-255

24. Pangan JC, Estrade R, Rosales R: Cholecystoduodenocolic fistula with recurrent gallstone ileus.Arch Surg 1984; 119:1201-1203

25. Cosio FG, Onstad GR: Pancreatic pseudocyst communicating with both the duodenum and colon. Am J Gastroenterol 1972; 57: 353-358 